\title{
LA COSECHA COMO SOPORTE
}

\author{
José López-Canti y FÉlix de la Iglesia Salgado \\ Departamento de Proyectos Arquitectonicos. Universidad de Sevilla
}

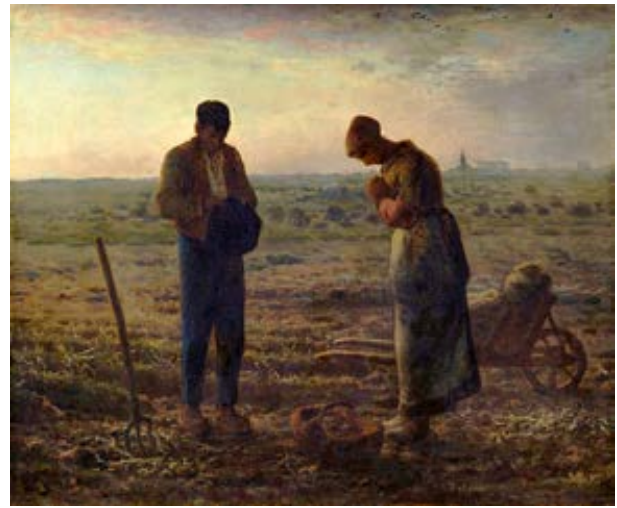

Fig.1 Jean François Millet_El Ángelus. 1859

«Combinar lo extraño con lo familiar: a eso aspiraba Ferguson, a observar el mundo tan detenidamente como el más entregado realista y sin embargo a crear una forma de ver la realidad a través de un prisma diferente, ligeramente deformante, (...) ylo que Ferguson pretendía por encima de todo era escribir historias que dejaran espacio no sólo al mundo visible de los seres sensibles y los objetos inanimados sino también a las vastasy misteriosas fuerzas invisibles que se ocultaban en el interior de lo ya visto» ${ }^{1}$

Con estas palabras de Auster se inicia lo que es el último episodio de un contrastado proceso de ejercitación en la docencia de Proyectos Arquitectónicos de la ETSA de Sevilla; un proceso

1 Paul Auster. “4, 3, 2, 1“. 2017 que, desde 2012, ha abordado el aprendizaje de las técnicas e instrumentos proyectuales con tanteos sucesivos localizados en el mundo agrario y sin unas referencias determinantes que nos llevaran de la mano a resultados previsibles. ¿Una búsqueda intuitiva? Se trata más bien de una deriva, apenas orquestada, a partir de dos consideraciones previas mantenidas en el tiempo: la primera, salir de Sevilla para situarnos en su corona metropolitana, en ámbitos caracterizados tanto por su valor medioambiental como por las dinámicas de ocupación de suelo, y con unos modos de vida que hoy no encuentran su sentido pleno en su condición periférica; la segunda, hacerlo bajo el prisma de la agr-y-cultura ${ }^{2}$, cambiando la

2 Expresión que encuentra en la cosecha, además del hecho en sí de la recolección del fruto que nos ofrece la tierra en sus distintos tiempos, la potencia del cultivar-se, del oficio y el saber, de la incertidumbre y la celebración... 
habitual mirada urbana por la agroecológica, de especial relevancia en estos momentos por su incidencia social y territorial al estar estrechamente vinculada a las pequeñas comunidades que los habitan, y como vector de renovación que activa un necesario equilibrio entre individuos y territorios.

Nos hemos desplazado, pues, a unas posiciones relativas desde las que mirar la ciudad y releer el mundo para actuar como individuos, como arquitectos: al espacio de los confines ${ }^{3}$ como región para la acción donde ensayar un acompañamiento que considere lo extraño y lo familiar, la mirada científica y la narrativa, que trate lo visible y lo invisible. Allí acudimos con una formatividad desveladora de geografías, marismas y campiñas, ciudades y campamentos, migraciones e inmigraciones, artistas y poetas, filósofos y pensadores, críticos y conformes, religiones y culturas,... haciendo del conjunto de esas lecturas reveladoras, guiadas por el azar de los relatos allí encontrados, una historia 'unificadora' que nos viene de su producción agraria característica y nos conduce a la cosecha como una infraestructura (espaciotemporal) que atiende tanto a la gran escala del campo de cultivo, a la vasta extensión superficial de un territorio familiar, como a la más íntima de los comportamientos, a la habitación como soporte de una habitabilidad y unos modos de existencia propios del sitio. Así el gar-

3 Dos referencias apoyan este enunciado. En una, "Confines", de Franco Rella, se nos sitúa en "nuestro presente, un territorio híbrido y ambiguo, indefinido y trágico. Un espacio especulativo que se acerca a la pluralidad y a las contradicciones de nuestro mundo y de los seres que la habitan. El espacio de los confines en la época en que todos los límites han saltado por los aires". La otra, "El Borde de una Ciudad", de Steven Holl, para quien 'el borde de una ciudad es una región filosófica en la que la ciudad y el paisaje natural se solapan, existiendo sin remedio ni expectativas'. banzo, el arroz, la oliva o, más recientemente, el tomate, lo inundan todo, haciendo de estas experiencias y lugares algo a la vez propio -asociado a nuestra vivencia cotidiana, tranquila, de afinidades y afectos- e impropio -derivado de los modos de producción y trato con la tierra-, lo que nos permitirá acudir a lo concreto de cada situación con el extrañamiento necesario para la innovación y la creatividad. El sitio, así, con sus culturas, sus escalas y tiempos, sus atmósferas, texturas y materialidad..., se convierte aquí en un personaje más con quien proponer y negociar otras formas de expresión y de lenguaje nacidas del susurro de las cosas y los moradores; y ha sido de este diálogo y compromiso con el medio ambiente y sus gentes como han surgido tanto nuevos sentidos como instrumentos para lo arquitectónico.

Estas 'artes de hacer', que podrían remitirnos al Michel de Certeau de La invención de lo cotidiano, se han venido contrastando simultáneamente con trabajos de investigación de posgrado sobre Permacultura y Agroecología, de tal manera que, para la edición del MARPH 2011, Stefania Scamardi señalaba en su Trabajo Fin de Máster ${ }^{4}$ la relevancia del discurso de la permacultura para el devenir de la vida en nuestras ciudades, construyendo con la imagen y la palabra una pequeña (gran) historia a través de detalles en apariencia insignificantes, de los enseres más cotidianos, de las huellas -tan dramáticas como esperanzadas- de la casa, del

4 Stefania Scamardi, "Sevilla celeste. Aproximación al paisaje vecinal de las azoteas del siglo XXI. Hacia la tutela de sus valores socio-ecológicos y culturales". 2012. TFM, cierre de la experiencia docente del Máster en Arquitectura y Patrimonio Histórico (MARPH 2011), a cuya exposición y defensa asistieron Luciano Furcas, referente local en permacultura (dinamizador del abandonado Huerto del Rey Moro, en el centro histórico de Sevilla) y representantes de movimientos ciudadanos. 


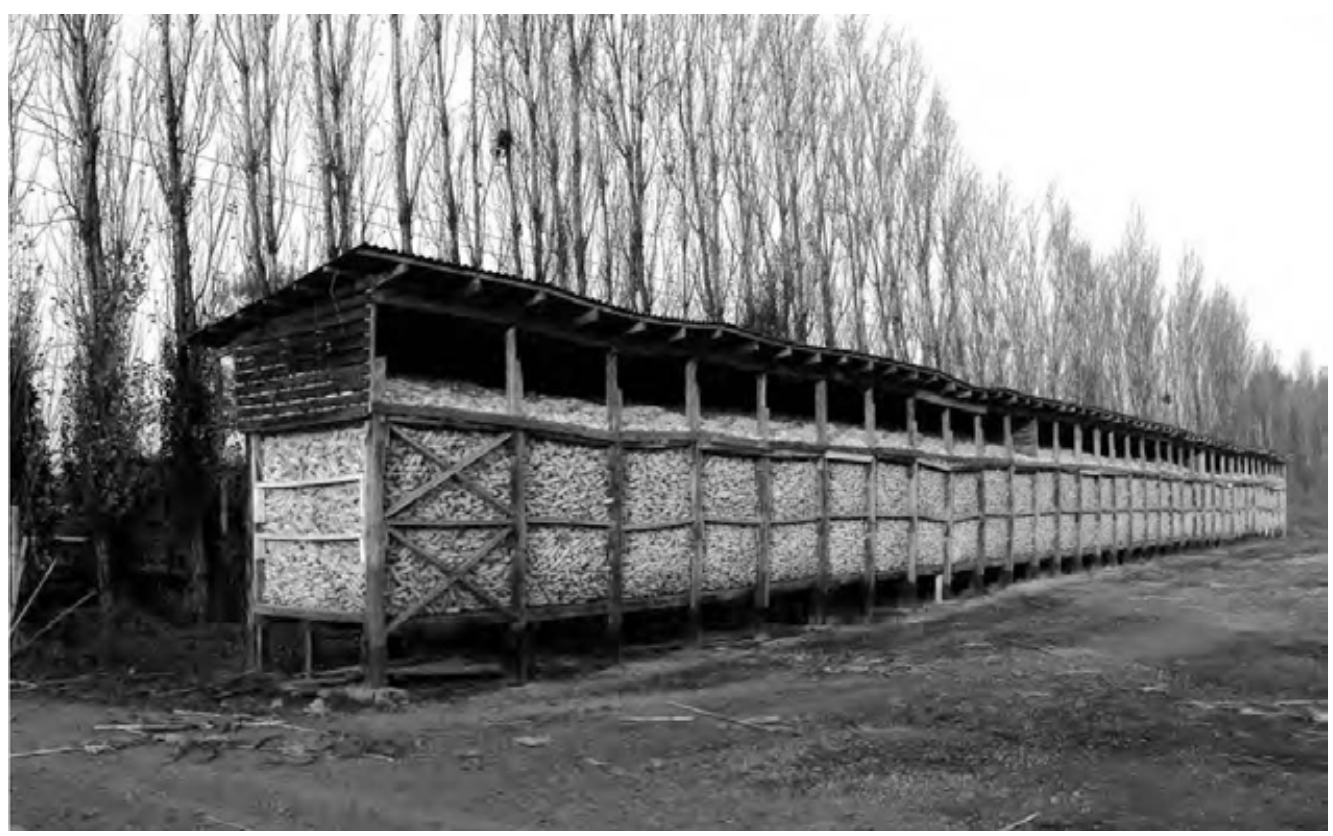

Fig. 2 Bodega de secado de maíz en Linares, Chile.2008. Fot. Manuel Gaete. En La contemporanización de lo vernáculo en la arquitectura: el caso del Valle Central de Chile, de José Luis Uribe Ortiz

trato cuidadoso de los habitantes con las cosas, de los 'cuasi objetos' que nos sitúan entre la tierra y el cielo, dando que pensar sobre el papel que jugamos cuando intervenimos en estas experiencias que se instalan en lo ecológico y lo cultural. Ya en 2013, en el marco del MCAS 2012, Marianna Papapietro se proponía como objetivo de su trabajo de investigación ${ }^{5}$ "redefinir la relación entre lo urbano y lo rural en las áreas metropolitanas, buscando el equilibrio territorial, respetando la vocación de cada área y la diversidad de usos, hábitats, paisajes y culturas que albergan". Y entre otras aportaciones, recogía dos

5 Maria Anna Papapietro, "Criterios de intervención en el paisaje de transición urbano/rural. El papel de la agroecología". 2013. TFM del Máster en Ciudad y Arquitectura Sostenibles (MCAS 2012). En https://issuu.com/mariannapapapietro/ docs/criterio_paisaje_urbano-rural_agroe. Visitado en diciembre 2019. referencias que nos interesaron especialmente: la primera, de Daniel López y Mireia Llorente (La agroecología: hacia un nuevo modelo agrario. Madrid, 2010), venía a decir que "la agroecología propone formas de desarrollo rural sostenible basadas en la unión de los conocimientos campesinos tradicionales de cada lugar y las aportaciones del conocimiento científico moderno, junto con el fortalecimiento de las redes sociales y económicas locales. Por otro lado, desarrolla técnicas de manejo agrario basada en la recuperación de la fertilidad del suelo, el aumento de la biodiversidad y el uso eficiente de los recursos disponibles"; y una segunda, de Roberto P. Guimaraes (Fundamentos territoriales y bioregionales de la planificación. Santiago de Chile 2001), para quien "la biorregión es aquel territorio, delimitado por límites blandos, que conforma un espacio con características naturales, 


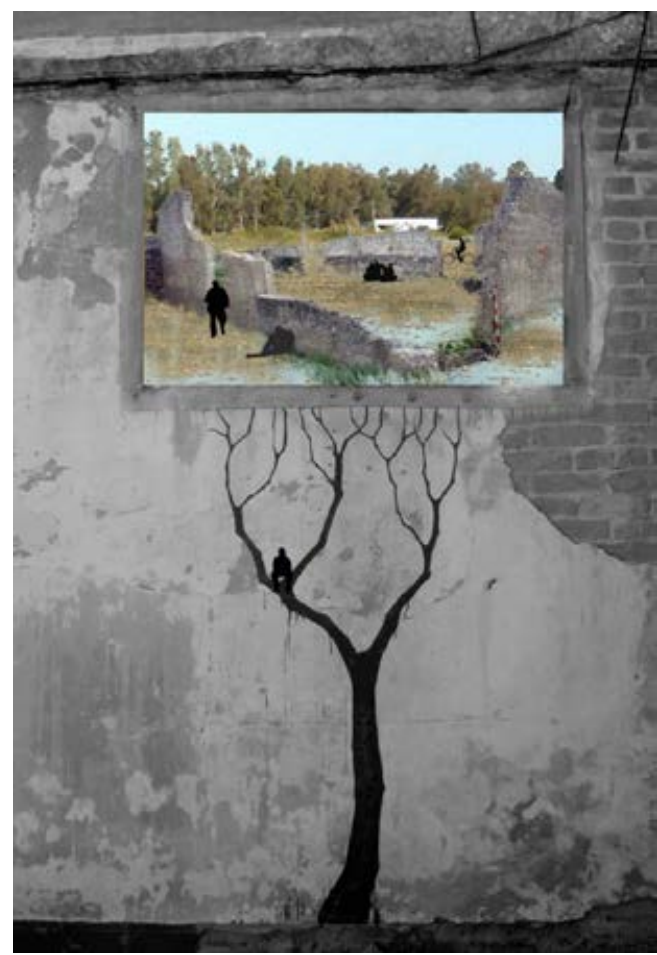

Fig. 3 Propuesta en la aldea Queipo de Llano. María Herrera y Ana Paniagua. 2014 grupo 12

geográficas, históricas y económicas comunes, y que potencia sus valores identitarios. Las biorregiones son una experiencia social y natural, y son conciencia que surge de relacionarse a través del tiempo con el terreno y el paisaje en particular. En tanto que mucha de la conciencia humana es atraída hacia un mundo global cada vez más abstracto, la visión biorregional busca volver a tejer la red de las relaciones entre la gente y el lugar". Ambos trabajos de investigación de las estudiantes de posgrado fueron, en gran medida, reflejo y activadores de una sensibilidad que por aquel entonces era aún incipiente y minoritaria en nuestro espacio docente. Desde entonces, en ello estamos ocupados: en recomponer un nuevo y distinto equilibrio entre lo urbano y la naturaleza que lo envuelve como punto de partida para fijar unos mínimos principios de aproximación a su solución, pero sobre todo para ensayar otras lógicas y maneras de hacer de lo arquitectónico para con la habitabilidad contemporánea.

En este tiempo hemos trabajado en el silencio de los vacíos desvelados teniendo la oportunidad de valorar lo existente, lo ambiental y lo agrario; preocupados por el desarraigo de tantos o el sinsentido de los soportes habitacionales desocupados que empiezan a ser un lugar común en los tiempos que vivimos, pero al margen de la presión envolvente que caracteriza a la ciudad cuando el consumo y el desarrollo parecen invadirlo todo con sus modos de vida, con su visión especulativa y depredadora. Y hemos podido verlos como lugares de cohabitación cargados de simbolismo, de materialidad y proyección participada: puertos de una aldea global, pantalanes abiertos al acogimiento de lo que proviene de fuera y que, con su recepción, nos llevan a una revisión de nuestras metodologías de aprendizaje y a ensayar nuevas herramientas para ser más eficaces. A partir de esta construcción de sentido, se configura un tablero de juego donde se hacen explícitos los materiales de una habitabilidad propia del sitio, donde somos capaces de señalar los acontecimientos, elementos y comportamientos que establecen este acuerdo singular entre mundo y vida, a la vez que se plantean nuevas ejercitaciones técnicas. La incertidumbre y el riesgo, lo necesario o contingente, el paisaje y la población con sus relatos, la inmensidad de los lugares o los microespacios singulares..., dan pie a detenerse en propuestas tradicionales $y$, en muchos casos olvidadas, para ser activadas como herramientas de proyecto. 


\section{AGROTRILOGÍA}

Queremos relatar aquí a modo de memoria lejana, unas experiencias docentes realizadas a lo largo de varios años con estudiantes de la Escuela de Arquitectura, del primer curso en la materia de proyectos arquitectónicos, y que tienen como foco de atención el territorio de la agricultura, el espacio agrario. Llama la atención de inmediato que, si se trata de construir, realizar proyectos y modificaciones de la realidad, el escenario resulta especialmente excéntrico, por su clara oposición al conglomerado urbano, por su estructura mínimamente urbanizada -apenas en términos de estricta necesidad-, y por la borrosa visión que un estudiante de arquitectura pudiera tener en esos escenarios como campo de acción. El desdibujo de los límites disciplinares de la propia enseñanza del proyecto, una vocación de creciente transversalidad y un contacto con diversas actividades que ahora creemos de especial importancia para la arquitectura pueden estar detrás de una razonable explicación al por qué de estos escenarios ciertamente antiurbanos. En efecto, el interiorismo -más allá del diseño interior-, como referente productor de habitabilidades y pilar de la autogestión de ambientes espaciales privados, las ciencias ambientales, como otro campo alternativo de explicación post-urbanística de las cualidades del territorio, la gestión del tiempo social, la administración del ocio o los aspectos de salud pública que impactan en una suerte de tela de araña sobre el registro de nuestra biografía espacial y sus derivas, son sólo algunos de los elementos que animan a la elección de estas configuraciones agrarias como campo y laboratorio docente de cara a la iniciación en el proyecto.

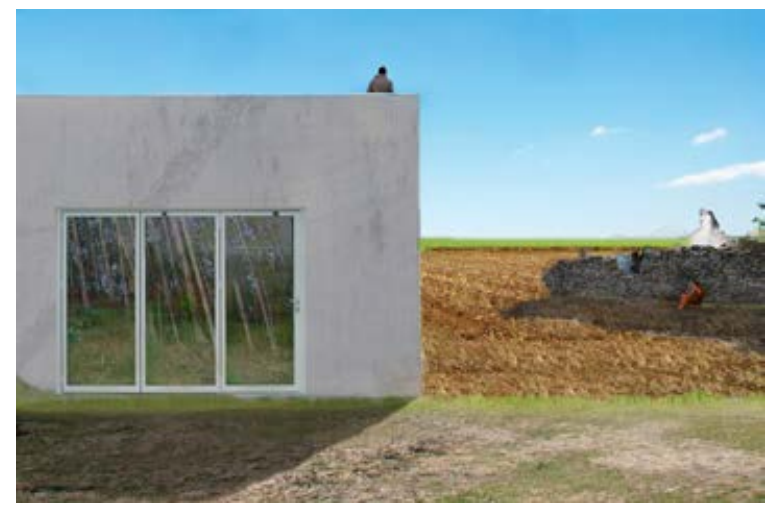

Fig. 4 Propuesta en la aldea Queipo de Llano. María Herrera y Ana Paniagua. 2014 grupo 12

Unos de los escenarios visitados y ejercitados -dentro de lo que vendrá a constituir una suerte de trilogía- habrá sido el del arroz situado en el entorno de la provincia de Sevilla. Una de las grandes regiones productoras dentro de país, en términos de superficie, muy próxima y accesible desde el término de la propia Sevilla, que a su vez está bastante virada dentro de la representación en el imaginario ciudadano, y existe literalmente de espaldas a su conciencia. Íntimamente ligada a la marisma, al devenir de las últimas decenas de kilómetros del Guadalquivir en su desembocadura atlántica y en clara colindancia con el parque nacional de Doñana, sus condiciones le aseguran rentabilidad anticipada como escenografía docente. No existe pues este registro territorial sin un enlace con la Sevilla región metropolitana, pero tampoco sin las continuas acciones que sobre el río hace el imput económico de la autoridad portuaria, $y$ las distintas tensiones que el necesario régimen hídrico que demanda la propia personalidad del cultivo, y que a su vez acaban implicando tensión con la gestión del propio parque nacional supeditado a un rango jurídico de régimen y al- 


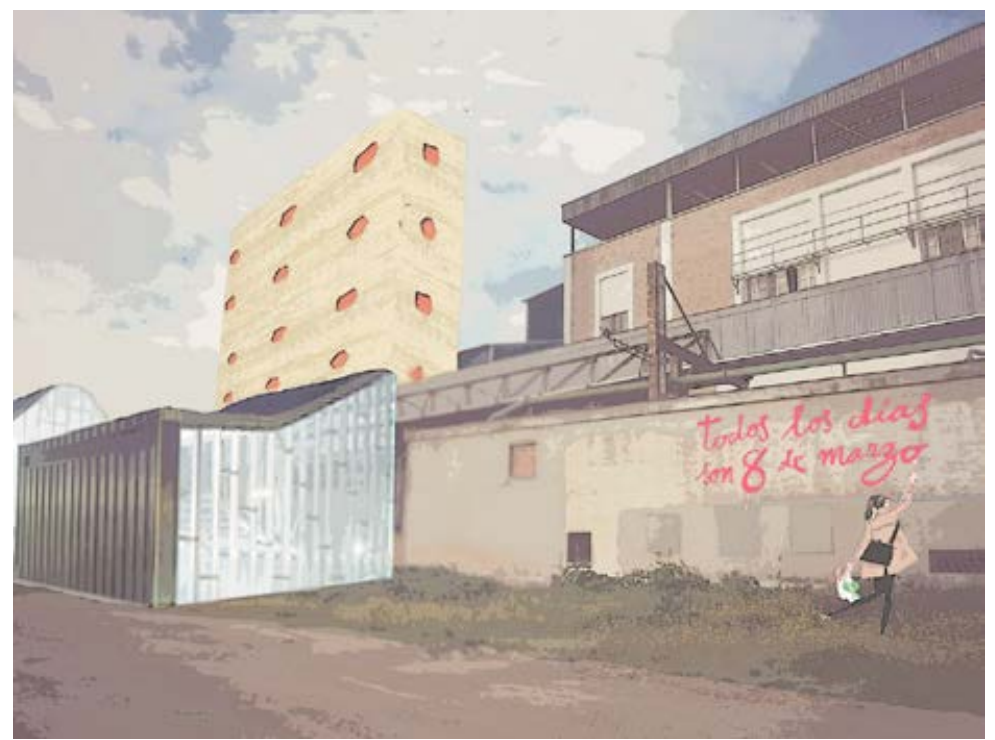

Fig. 5 Propuesta en la arrocera de San Juan. Ángela Albero y Patricia Sánchez. 2014 grupo 05a

cance europeo. Casi todas las escalas pues, de la gestión y del territorio, de sus actividades y elementos naturales, se dan cita en un espacio extraordinariamente plano, pero excesivamente rugoso en superficie, por lo complejo de su composición y lo enrevesado de su accesibilidad interpretativa. El espacio agrario finalmente, es complementado con una de las instalaciones más importantes de recogida, almacenamiento, transformación, envasado y distribución ubicada en la localidad de San Juan de Aznalfarache -prácticamente soldada a la idea de Sevilla como ciudad-, por lo que el ciclo pretende ser comprendido en su dimensión más completa posible. La primera aportación como ventaja que se deriva de este tipo de situaciones y la tornan un laboratorio docente, es la notable ausencia de arquitecturas tal como las entendemos en el soporte de lo urbano y la ciudad. Bien es cierto que todo un conjunto antrópico de huellas plaga estos territorios, de canales y canalizaciones, de máquinas y estaciones de bombeo, de casetas y pequeños municipios que, subrogados a la actividad agrícola, se manifiestan aún hoy en día con una austeridad vital y una dificultad con el ambiente en el que anida la memoria del pasado como un territorio de convictos fugados, de rechazados por su propia voluntad o por las difíciles condiciones históricas del momento. Historias de colonización, siempre puestas en sospecha por la obligatoriedad o voluntariedad del movimiento migratorio, en la que la extensión y llanura del territorio devora cualquier gesto arquitectónico. Lo agrícola es un territorio que describe con mucha fiabilidad el devenir de la historia, a pesar de la aparente fungibilidad de las cosechas, y su retorno periódico; las huellas suelen perdurar sin eliminarse en sucesivos palimpsestos, más bien siempre se muestran disponibles como ruinas abandonadas, en las que la demolición es más costosa que lo que acaba destruyendo el tiempo: tal es el caso del poblado que lleva el nombre del general rebelde Queipo de Llano, y cuya poquísima importancia aún imaginándolo recién inaugurado, deja ver a las claras las tensiones en el interior del propio régimen franquista: estas huellas se hacen especialmente nítidas en este soporte de la agricultura. No en vano, la mayoría de bustos romanos, de estatuas mutiladas, de fustes y capiteles que hoy ocupan espacios de museos y casas palaciegas de grandes terratenientes 
proceden de los eventuales tropiezos antaño de las herramientas de arado sobre la superficie agrícola de los campos andaluces. La historia sobre el soporte agrícola siempre se constituye en contemporánea y aquello que deja de funcionar, las más de las veces se constituye en una gran maceta de la que brotan uno o dos árboles antiguos del interior de lo que debió ser algo cubierto y habitado. Son pues estos debilísimos argumentos de modelación del territorio los escasos acompañantes de los estudiantes; caminos sin asfaltar, perspectivas infinitas modeladas por las espigas de arroz, un barco en plena navegación, muy a lo lejos, como si hendiese la tierra cual mar helado, postes eléctricos, cunetas, casetas y pequeños ingenios, como la distinguida película de $<<$ Isla mínima $>$ nos mostró cruzada de lleno con la narración, y ayudándose con frecuencia de la verticalidad ascendente del dron para poder ganar visión, perspectiva y descripción del asfixiante territorio laberíntico en todos los sentidos. Y la contingencia estacional, no sólo proveniente de la mudanza atmosférica, sino el propio ritmo y calendario al que sujeta el aspecto del cultivo: cosecha, siembra, inundación, brote, crecimiento, cosecha y preparación de la siguiente campaña, en un ritmo sisifoniano del tiempo, con un re-

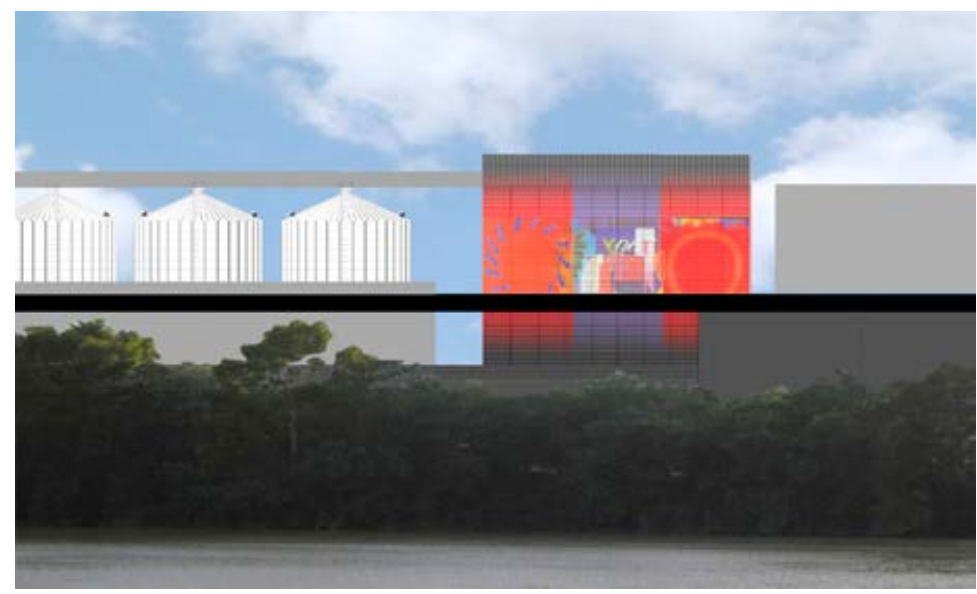

Fig. 6 Propuesta en la arrocera de San Juan. Ana Camacho y Eduardo Cuadrado. 2014 grupo 17

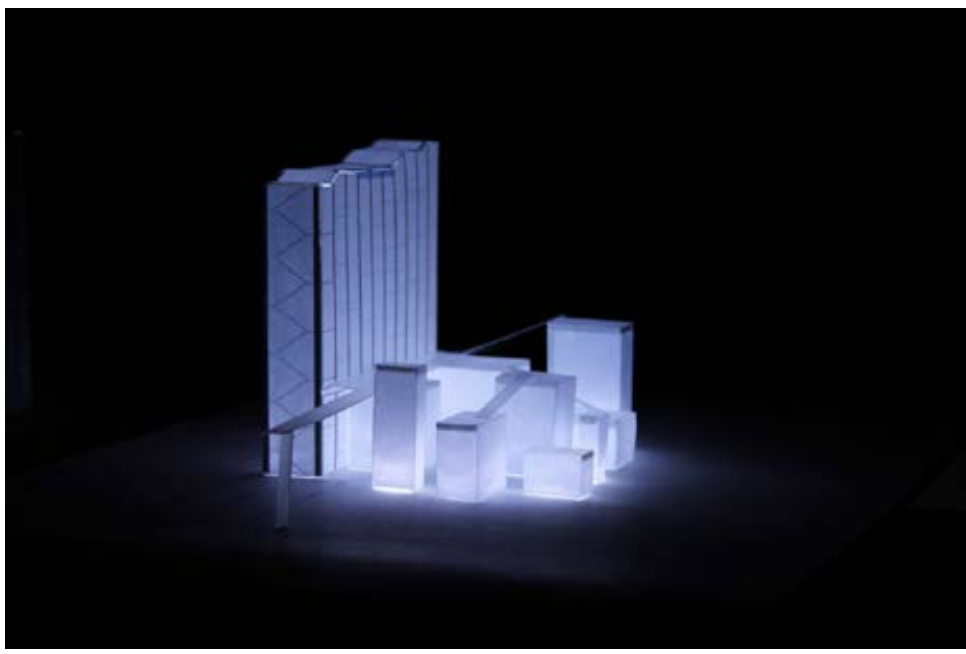

Fig. 7 Propuesta en la arrocera de San Juan. Andrés Galera y Mónica Castellanos. 2014 grupo $14 a$ gistro ambiental lleno de mudanza y repetición. Son pues estos paisajes corrientes de la producción agraria los que desinhiben de mejor manera aquellos prejuicios adquiridos en torno a la arquitectura, sus lenguajes y acciones propias, llevando al límite la economía del pensamiento 
y las emociones. El retorno a la industria transformadora de este proceso productivo, es quizá la parte más desconcertante de este devenir territorial. Allí, en San Juan de Aznalfarache y sobre la directa fachada del Guadalquivir instalaciones que se expresan en la lengua de lo industrial, pero también del almacenaje y los servicios, el laboratorio y la distribución, se reúne una cierta alquimia misteriosa de los mercados globales y las tensiones que el arroz como producto casi básico genera a lo largo del planeta. Es fundamental llegados a este punto del registro, diversificar y entrelazar las propuestas de los estudiantes para conseguir un enlace complejo con el espacio agrícola, y es ahí donde un programa de uso distinto al que aconseja la homogeneidad de la instalación y totalmente inopinado, hace su aparición, proponiendo la reconversión de uno de los silos de arroz en viviendas para mujeres que sufren maltrato $y$ violencia doméstica. Si la arquitectura se comportara como un ecosistema, esto significaría que su diversidad sería signo de fortaleza y resiliencia, y es justo la combinación y superposición de estos programas aparentemente incompatibles, los que añaden resistencia a las instalaciones contra las fluctuaciones económicas, el devenir del mercado y las frecuentes crisis. De este modo los estudiantes, a partir de la memoria agrícola del arroz, consiguen concentrar sobre la factoría de su procesado toda una serie de cualidades y memorias que proceden de los campos de cultivo próximos, donde una gestión del tiempo más circular, un contacto más directo con el ambiente desurbano y unas cualidades balsámicas de un paisaje singular al tiempo que corriente, se constituyen en terapia espacial que reconstruye relaciones y repara heridas inexplicables. Son estos engarces del territorio de lo uno hacia lo otro o, mejor dicho, hacia el otro, los que arman el discurso de una habitación llena de oportunidades que no se generaría sin el recurso del estudio y la relación con la tierra culta.

Otra de las experiencias en este devenir de trilogía que quisiéramos brevemente comentar, tiene que ver con la realizada sobre la geografía de Paterna del Campo y su entorno territorial -entre las que se incluyen las instalaciones patrimoniales del llamado Castillo de Alpizar- y que, en este caso, tienen como centralidad del cultivo el espacio del garbanzo y en un segundo plano, pero no menos identitario, la producción del aceite de oliva. Muy próximo a la extraordinaria cultura agrícola del espacio onubense del Condado, estos territorios son un auténtico reservorio de memoria histórica a partir de la fungibilidad de sus cultivos que le configuran un paisaje propio y característico. El garbanzo se caracteriza por la ausencia casi total de árboles en sus campos, casi como si este motivo facilitase y favoreciera la existencia del segundo; tierras arcillosas y expansivas, están detrás de la explicación que caracteriza esta llanura tan solitaria donde un producto con denominación hace sentirse orgullosa al conjunto de la población. Nuevamente son los restos patrimoniales, muy someros y espontáneos los que salpican directamente a la vista un devenir más que milenario, haciendo que la característica circularidad del tiempo campesino se complemente directamente con una veloz flecha que apunta hacia el pasado con una visibilidad nítida difícil, muy difícil, de encontrar y leer sobre el soporte urbano, dominado de forma sofocante por los siglos XIX y XX en un choque contemporáneo que hace sofocante en las ciudades la percepción ilimitadamente constante del tiempo presente y 
de la atmósfera contemporánea, a pesar de toda la irrupción del mundo virtual que concentran por naturaleza las habitaciones y calles de las ciudades. Rápidamente los estudiantes captan el sentido cooperativo de esta producción, la agregación de pequeños propietarios que sumando bajo una organización reglada hace que esfuerzos que en solitario difícilmente drenarían beneficios y permitirían supervivencia del modelo y continuidad en el tiempo, lo conviertan en posible, en éxito que supone cohesión e identidad, y casi como efecto de parentesco, reconocen

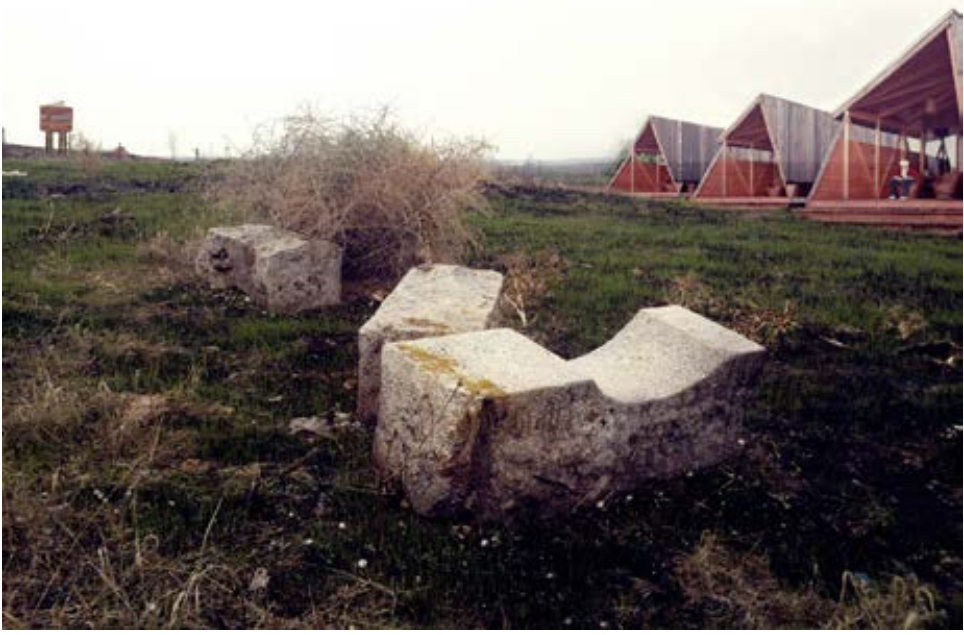

Fig. 8 Propuesta en el Castillo del Alpizar. Daniel Pérez y Manuel Silva. 2013 grupo 11 con facilidad que las tareas de la arquitectura sobre estas geografías y territorios han de ser abordada igualmente como trabajo colectivo y cooperante: los grupos de trabajo en los que desarrollan la actividad del proyecto evidencian, aunque sea por un principio de la cantidad, lo complejo y difícil que resulta un empeño en solitario. En estas localidades de vocación eminentemente agraria, los excedentes de tiempo se gestionan de forma muy distinta a la urbana (un cierto tiempo híbrido empieza ahora a ensayarse sobre el ámbito de las ciudades) y deja una notable huella sobre los territorios de inmediata proximidad a las localidades. Así, todo un conjunto de pequeñas huertas comunitarias, localizadas con inteligencia a en los terrenos más fértiles, de mayor proximidad al acceso sostenible del agua, hablan de una vida extendida más allá de la casa del pueblo, en franca proximidad pero que anhela esa fuga emocional que siempre suponen los espacios abiertos al territorio, don-

de las escalas chocan sin solución de continuidad en una situación de vértigo para la percepción de los estudiantes: reciclaje, pequeños ingenios, agrobricolage y, sobre todo, un moderado sentido hedonista de la vida, muy apegado a los productos de la tierra y sus sabores, pequeños placeres que suman en la economía familiar otro añadido extra modesto y cuasi medieval, que a la larga acaba permitiendo la permanencia de la población y la posibilidad de la cohesión social, justo ahora que se ha puesto en evidencia el concepto del territorio vaciado y en el que la fuerza de lo político se aproxima siempre con la negra sombra del oportunismo y la hipocresía. No es esta una sociedad subsidiada, como se ha dicho con carácter despreciativo las más de las veces, sino una clase social casi única cuya relación con la tierra y el territorio cada vez es más desconocido y más ignorada en la cultura urbana que todo lo cubre de artificio como necesidad 
imperiosa. El turismo rural es un aspecto más de esta especie de brecha de ignorancia que se trata de acortar con experiencias impostadas, con un imposible anhelo de autenticidad que brota con fuerza los fines de semana y las fiestas de guardar y que se extiende en pequeños puentes temporales. La sostenibilidad como tendencia también ha sido causante involuntariamente de parte de este desencuentro, al señalar sin dudar a las ciudades como los espacios de impacto de los desarreglos que hacen tambalear al conjunto del planeta; si bien esto no falta a la verdad, no es toda la verdad, puesto que no ha articulado ningún discurso con el resto de la estructura territorial entre las que se encuentran los espacios que estamos reseñando. La necesidad en relación a lo agrícola de aproximar la distancia -tanto material como mental- al borroso límite las ciudades, manifestándose en el consumo de proximidad, en los grupos de consumo de libre asociación, son todas ellas estrategias que quieren ahuyentar las grandes distancias que los productos agrícolas recorren hasta nuestros platos, la falta de sincronía entre la temporada natural de los productos, y su disponibilidad artificiosa a lo largo del año, creando una insatisfacción en su calidad, sabor y, si pudiéramos expresarlo así, su autenticidad. Esto sin género de dudas ofrece una oportunidad única de reorganizar las economías regionales, sus costumbres y hábitos saludables, en una suerte de biorregión, unidad que parece organizada desde condiciones más sensatas que los parámetros tradiciones que han operado en los últimos decenios sobre la organización territorial y administrativa del espacio que habitamos. De ahí es donde podríamos concluir que tanto el arroz como el garbanzo están en franca proximidad con el área metropolitana de Sevilla.
Quisiéramos finalmente incorporar otro elemento que concluye esta rápida trilogía, y que no podrá ser detallada con precisión debido a que justo en estos momentos transcurre como actividad docente aún inconclusa. Nos referimos al fenómeno socioespacial que constituyen los llamados 'plástico' en la provincia oriental de Almería; sus más de 500 kilómetros cuadros, que sin solución de continuidad llenas la geografía con una densidad desconocida hasta el momento; como se acostumbra a decir, para ser gráficos, la visibilidad que el fenómeno tiene desde la exterioridad del planeta -como fácilmente se podrá verificar en la aplicación de Google Earth-, o la ligera disminución de la temperatura media regional como consecuencia de la enorme superficie reflectante que devuelve parte de la radiación solar, son sólo algunos datos que aproximan a la mente la escala del fenómeno que estamos relatando. El abordaje para los estudiantes es francamente complejo; existe una primera reacción comprensiva que consiste en la paralización, el pensamiento no logra sincronizarse con lo visto. Sin embargo, las oportunidades que se ofrecen en términos de experimentación y aprendizaje se constituyen en únicas. No es el lugar ni el momento de analizar el fenómeno en profundidad, apenas señalizar algunos aspectos que nos parecen imprescindibles y que acaban constituyéndose en dudas más que razonables para el visitante foráneo del fenómeno. En primer lugar, reconocer esta transformación gigante como el esfuerzo sincronizado de toda una sociedad, cuasi al unísono, que en llegados al presente medio siglo después, ha obligado a las administraciones a poner freno en el fenómeno por su impacto 
de uso y consumo de suelo en el territorio. finalmente, una reconsideración a lo que llaDistinguir en segunda instancia, explotacio- mamos agricultura ecológica: no sólo puede nes o invernaderos de carácter tradicional, recaer esta certificación entre los producido de aquéllos otros de gran escala y tecnifica- y los productos necesarios para producirlo; ción que han llegado a un procedimiento de otras consideraciones, como el impacto teabstracción tal sobre la producción, que los cultivos ya no están vinculados a la tierra sobre la que se sitúan. Bolsas aéreas con todo lo necesario para el crecimiento, producen este corte umbilical que desde luego reescribe la historia de la agricultura y la proyecta hacia una dimensión virtual. La deslocalización y la desterritorialización quedan servidas en bandeja, como se expresa vulgarmente. $Y$ rritorial, el consumo de energía, la identidad geográfica y la gestión del paisaje -incluso a su nivel de condición corriente- son aspectos que sufren indeciblemente a pesar de la garantía ecología de lo producido. No cabe duda que también se percibe una densidad ínfima de mano de obra, a veces evaluada entre 2-3 personas por hectárea, lo que lo convierte por veces en naves fantasmas y silenciosas que

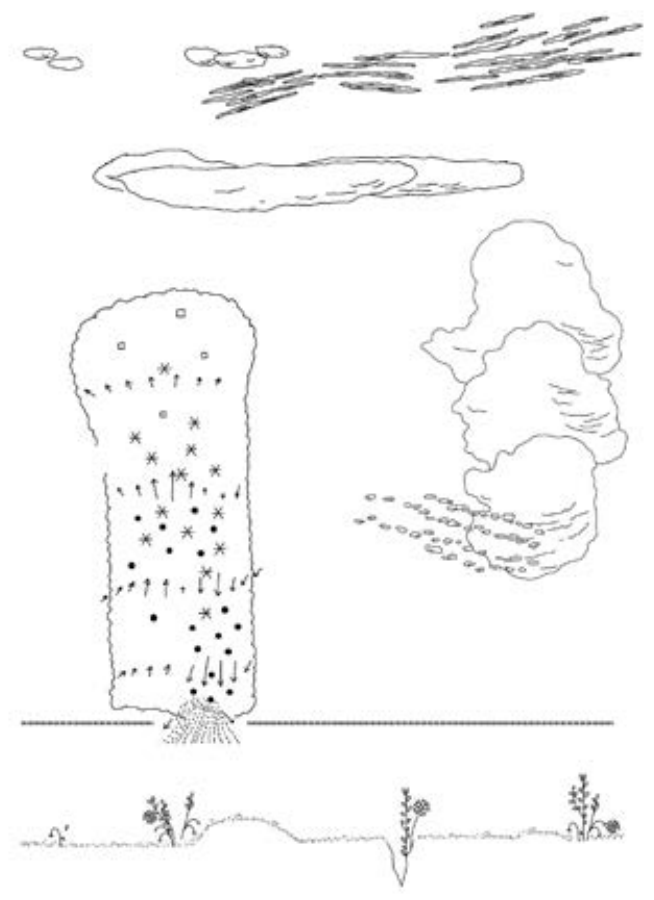

Fig. 9 Paula Fernández San Marcos. TFM Paisajes Corrientes. Cartografía del Archipiélago. 2019 
ignoran su ubicación en el mundo. En términos de acción proyectual la terea es infinita y dilatada, tanto como el propio mar de plásticos sobre la que se extiende. La regeneración de espacios públicos que esponjen y aireen la densidad sofocante de los invernaderos, y promuevan un soporte de posibilidad de relación, convivencia y ocio; la revisión global de la habitabilidad de la región, en convivencia con el estilo del cultivo, que mejore la mala calidad urbana y los pésimos estándares que se adivinan, faltos de articulación y mínimas armonías, teniendo muy presente la enorme colonia de inmigración -de mayoría subsahariana- cuya calidad de vida y especialmente de habitación, deja mucho que desear, y cuya articulación política se adivina llena de tensión y ausencia de integración, son todas ellas tareas urgentes y de primera necesidad donde el estudiante no hace articulaciones abstractas, sino combinaciones con un estilo de cultivo capaz incluso de borrar el Mediterráneo -y todo lo que supone como reservorio cultural de habitabilidad-como elemento orientador de los proyectos. Situación compleja de la que esperamos dar nuevas noticias en otras ocasiones.

\section{PROGRAMACIÓN DE UN AMBIENTE: DE LA METRÓPOLI DE LA MENTE A LA BIORREGIÓN DE COMUNIDADES}

\author{
«La idea de 'metrópoli' no posee ya \\ ningún aspecto dimensional. 'Metró- \\ poli' es tanto todo el planeta como mi \\ cerebro. El viaje por los meandros de la \\ Tierra es idealmente idéntico al viaje \\ alrededor de mi habitación. Yésta me
}

parece, sin duda, una posibilidad de libertad como ninguna otra época ha conocido jamás» ${ }^{6}$

La experiencia docente, que en gran medida justifica este texto, se inserta en una preocupación de años por facilitar, tanto con las técnicas propias de la arquitectura como con las de otras referencias transversales, un acuerdo que respete la vocación y la diversidad de usos propios del medio rural y el urbano, especialmente en sus ámbitos de encuentro, y un soporte habitacional para las comunidades que allí desarrollan su existencia. Una actividad relevante por su incidencia social y ambiental tanto para el conocimiento, interpretación y valoración de estos sitios, como para la intervención y la gestión de los recursos y patrimonios contemporáneos. Plantear un distinto equilibrio entre lo urbano y su envolvente cuasi natural que respete la vocación y la diversidad de usos de cada uno de ellos, es un punto de partida para fijar unos mínimos principios de aproximación a su cohesión social y territorial, pero también y, sobre todo, para dimensionar el papel de la arquitectura y ensayar otras prácticas en relación a la habitabilidad contemporánea.

6 MASSIMO CACCIARI, "Metropoli della mente". Rev. Casabella, n 523 (1986). Con estas palabras nos conmovió Massimo Cacciari cuando en 1986 publicara "Metropoli della mente", poco tiempo después de que Bernardo Secchi nos propusiera en "Las condiciones han cambiado", 1984, tomar otro punto de vista, más periférico, para ver la ciudad; o antes, en el 62 y editado por Tusquets en 1991, Italo Calvino nos ayudara a releer el mundo con "El camino de San Giovanni": unas y otras lecturas dieron cuenta de lo necesario de nuevas interpretaciones que señalaran el valor de lo singular de determinados territorios locales para una situación que ya era global. 30 años después todos conocemos la inercia imparable de los procesos de ocupación del territorio y sus efectos en la sociedad: de la desafección a la exclusión, del monocultivo a la obsolescencia..., y muchos seguimos preocupados. 
Hoy, seguimos pretendiendo un conocimiento y una escritura que emane de estos territorios de proximidad y que, en un horizonte cercano, querríamos caracterizar como territorios en Transición, especialmente como lo formula Juan del Río7: "El concepto de transición parte del intento de aplicar el diseño de la permacultura a asentamientos y ciudades teniendo como pilares básicos a nivel conceptual el pico de producción del petróleo, el cambio climático y, para hacer frente a esos dos fenómenos interrelacionados, la resiliencia () La obligada vuelta a la pequeña escala debe verse en todo momento, no como un proceso de aislamiento, sino como un conocimiento entre comunidades y naciones que intentan ayudarse para incrementar su resiliencia dejando atrás una dependencia compartida". Con estas inmersiones en los márgenes y lo agrario queremos ejercitarnos con una lógica más cercana a la de la permacultura, a la de la biorregión, que a la urbana, como rechazo a un desarrollo que se nos hace ver como inevitable para garantizar el logrado estado del bienestar, a pesar de su alto impacto sobre el medio ambiente, y que cada vez más se aleja de un buen vivir justo y accesible para todos en el que la equidad se establezca como sentido de lo común para alcanzar su máximo potencial. Así, con esta mirada atenta a la justicia social, conscientes de estar en punto de no retorno y sabiendo que no hay una solución global que lo resuelva, el deseo de recomponer y actualizar la cadena de relaciones que ha propiciado los distintos equilibrios de este espacio relacional nos conduce a procesos de renaturali-

7 JUAN DEL RÍO. "De la idea a la acción; Aprendiendo del movimiento Transition Towns". 2009. Disponible en https:// upcommons.upc.edu/bitstream/handle/2099.1/8807/ Juan\%20del\%20R\%c3\%ado.pdf?sequence=1\&isAllowed=y zación ${ }^{8}$, localizados en ámbitos singulares de escalas, tiempos y condiciones diversas, procesos que inevitablemente asociaremos a la equidad si queremos que sean operativos y posibiliten otras políticas e instrumentos para comunidades específicas no referenciadas en la normalización y en las divisiones administrativas.

Una línea de trabajo que ha supuesto una lectura original y multiescalar de situaciones, fenómenos, asentamientos humanos, escenarios, de estrategias, técnicas y tecnologías que se mueven entre lo empírico y lo científico para dar cuenta de una doble mirada que abarca desde la visión territorial y patrimonial, hasta la percepción cercana de lo material y su vivencia. Desarrollada en diversas situaciones de encuentro y tránsito, en ecotonos nos dirían los biólogos, hemos navegado por estos espacios relacionales con la ayuda tanto de los documentos científicos disponibles como de los relatos hallados de sujetos fronterizos o la ficción de personajes libertarios. Una navegación entre enclaves de una red espacio-temporal que los reúne según sus afinidades y donde cada nodo, autónomo, se convierte en una posibilidad razonable de guiar decisiones y producir actividades compatibles con un medio cada vez más debilitado.

El desarrollo del trabajo perfila lo que se encierra hoy tras la idea de sostenibilidad (eficiencia y resiliencia de los territorios y las comunidades) fijando posiciones en el panorama cultural en general y en el pensamiento arquitectónico, en particular. Y ello ha sido po-

8 "A pesar de su vocación de interrupción y de evocación de un tiempo anterior, no se trata de alcanzar una completa reversión de los procesos antrópicos, sino de una ralentización o decrecimiento en el consumo de los recursos, promulgando la equidad". Ver Wikipedia: https://es.wikipedia.org/wiki/ Renaturalizaci\%C3\%B3n. Visitado en diciembre 2019. 
sible porque se ha acometido atendiendo a los asuntos de la vida, del mundo y sus representaciones, para configurar aquellos cosmogramas o soportes que -como visualización e instrumentación de la imagen- avanzaran hacia las intervenciones parciales o valoraran la gestión y administración de los recursos disponibles; contando con la la Red de actores, a la manera del "Victims" de Hejduk, haciéndola visible desde los pequeños relatos, los deseos y movimientos, para generar los acompañamientos que configurarían los escenarios de encuentros; disponiendo de los aparatos de intermediación necesarios, 'rehaciendo las imaginaciones' , para aprender de los acontecimientos; propiciando nuevos instrumentos, a la manera de diagramas, para la notación de los escenarios de encuentro. Es lo que hemos aprendido de estas figuras: la idoneidad de estos territorios para configurar un distinto imaginario en relación a la habitabilidad contemporánea y el alto potencial innovador para una arquitectura necesaria.

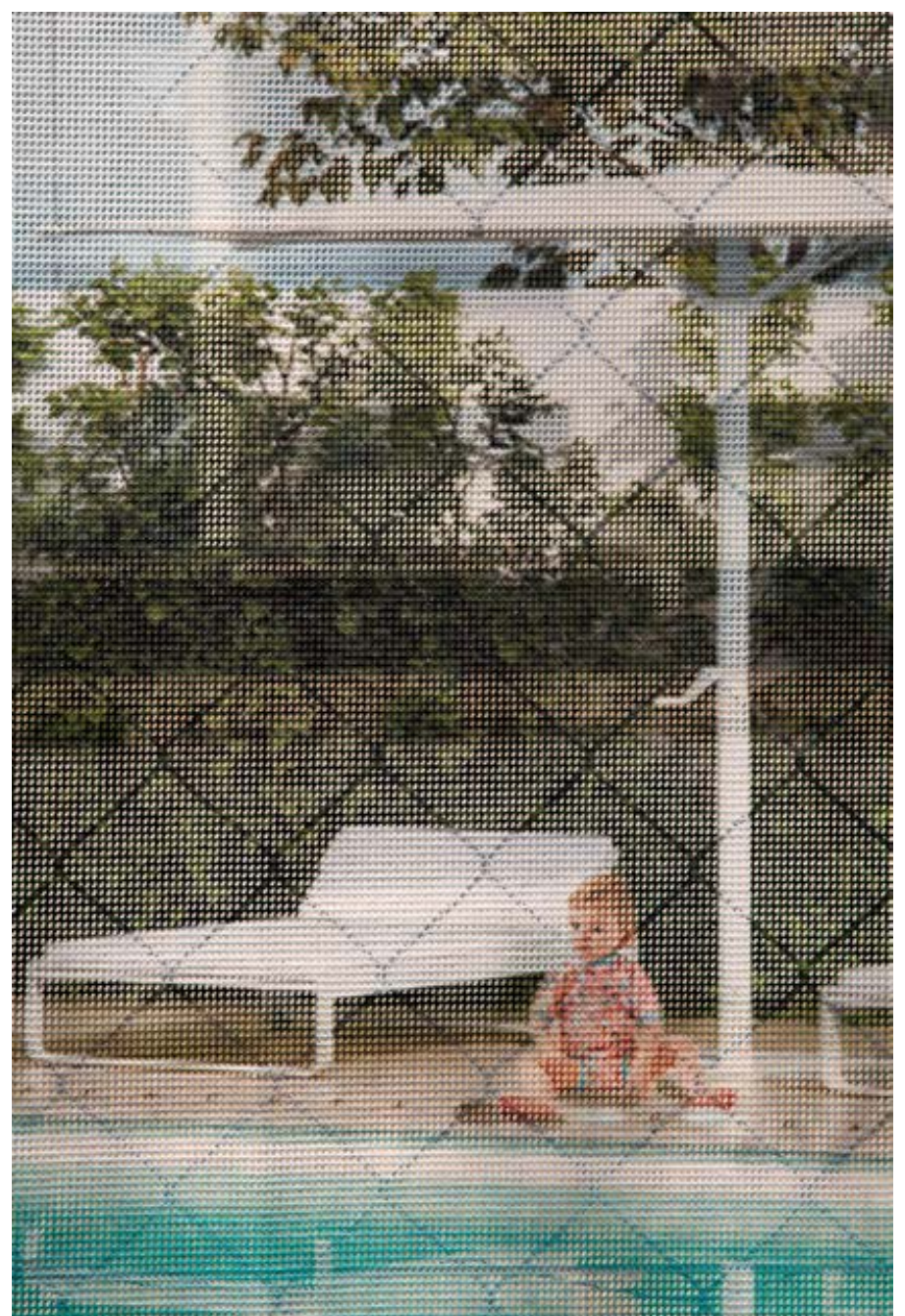

